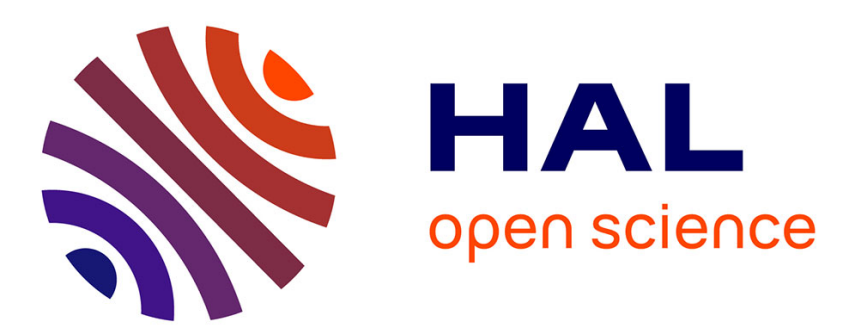

\title{
A robust CRE-based approach for model updating using in situ measurements
}

Pierre Etienne Charbonnel, Pierre Ladevèze, François Louf, Ch. Le Noac'h

\section{To cite this version:}

Pierre Etienne Charbonnel, Pierre Ladevèze, François Louf, Ch. Le Noac'h. A robust CRE-based approach for model updating using in situ measurements. Computers \& Structures, 2013, 129, pp.6373. 10.1016/j.compstruc.2013.08.002 . hal-01647864

\section{HAL Id: hal-01647864 \\ https://hal.science/hal-01647864}

Submitted on 17 Dec 2019

HAL is a multi-disciplinary open access archive for the deposit and dissemination of scientific research documents, whether they are published or not. The documents may come from teaching and research institutions in France or abroad, or from public or private research centers.
L'archive ouverte pluridisciplinaire HAL, est destinée au dépôt et à la diffusion de documents scientifiques de niveau recherche, publiés ou non, émanant des établissements d'enseignement et de recherche français ou étrangers, des laboratoires publics ou privés. 


\title{
A robust CRE-based approach for model updating using in situ measurements
}

\author{
P.-É. Charbonnel ${ }^{a, c, *}$, P. Ladevèze ${ }^{a}$, F. Louf $^{a}$, Ch. Le Noac'h ${ }^{b}$ \\ a LMT-Cachan, 61 avenue du Président Wilson, 94235 Cachan cedex, France \\ ${ }^{\mathrm{b}}$ ASTRIUM-ST, 66 route de Verneuil, 78130 Les Mureaux, France

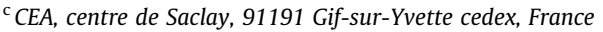

\begin{abstract}
This paper presents a systematic approach for comparing a numerical model to test results performed in situ on a structure with time-dependent behavior. A crucial issue for an engineer is to be able to assess the quality of models, based on a series of measurements. Dealing with in situ measurements as experimental reference for model updating involves two major difficulties: the excitations can be multiple and affected by large disturbances. The validation process we propose is based on the mechanical concept of Constitutive Relation Error (CRE) and aims at reducing the Lack Of Knowledge (LOK) attached to both the excitation forces and the parameters of the numerical model. The updated values can be computed inside confidence intervals that correspond to the lower contours of the CRE-based residual to minimize.

The proposed method will be illustrated with a numerical example taken from the aerospace industry and applied for correcting a simple ARIANE 5 model by comparison with flight measurements.
\end{abstract}

\section{Introduction}

The constant increase in computer resources and the considerable progress made in the field of modeling have enabled the simulation of very complex systems. Finite Element Modeling (FEM), in particular, provides for industry a systematic analysis tool, adapted to a wide range of problems. Of course, mastering the models is an essential requirement and in most cases, experimental validation is the best way to ensure that the response of the model is consistent with that of the actual structure.

This work focuses on validation or model updating; more precisely, its purpose is to derive a robust strategy, based on the Constitutive Relation Error (CRE) method for comparing a (possibly time dependent) FE model to a set of experimental measurements, performed in situ on a structure in its functional environment. The underlying objective using in situ measurements for validation, is to be able to qualify the models while avoiding duplicative experimental campaigns, which in addition to being expensive, only partially represent the operational conditions experienced by the actual structure (boundary conditions, level of excitation, etc.). Extensive literature reviews of validation/model updating approaches are provided in references [1-3]. The different methods described therein may be divided into two families.'Direct' methods propose to correct the FE matrices at once, through a

* Corresponding author at: CEA, centre de Saclay, 91191 Gif-sur-Yvette cedex, France. Tel.: +33 670367631; fax: +33 169088331.

E-mail address: pierreetienne.charbonnel@cea.fr (P.-É. Charbonnel). non-iterative process; those can be broadly categorized into different classes. Optimal Matrix Updates methods form a first class and aim at determining stiffness and mass matrices satisfying a set of measurements as well as verifying some mathematical properties [4-9]. Control-based Eigenstructure Assignment Techniques form a second class of methods and seek to determine the pseudo-control that would be required to produce the measured modal properties using the initial structural model [10-12]. The pseudo-control is then translated into matrix adjustments and applied to the initial FEM. A third class of non-iterative model registration methods uses the Minimum Rank Perturbation Theory where the update to each matrix is of minimum rank, equal to the number of experimentally measured modes that the modified model is to match [13-16]. Although this approach has proven computationally efficient for structural health monitoring and modal correlation, it does not allow to question the physical parameters of a given family of models and can lead to corrections which lack physical meaning.

The need to preserve the physical meaning of the corrections has led to the emergence of 'indirect' or parametric methods. The approach consists of building a cost function which represents the correlation between the numerical model and the test data; the changes in the stiffness and mass matrices are based on variations of the model's physical parameters. In contrast with direct methods, special attention must be paid to the preliminary selection of the parameters to update (as stressed e.g. in [17]); the scatter in the solution is intrinsic to the inverse problem for which multiple solutions may coexist [18,19]. Several types of cost functions can be used. They can be classified into three categories 
involving respectively input residuals [20,21], output residuals $[22,23]$ and energy-based residuals.

The validation method developed at LMT-CACHAN belongs to this last category. This approach consists of minimizing a parameterdependent cost function based on the concept of Constitutive Relation Error, which is a meaningful energy-based indicator. This posteriori estimator has been initially introduced for the purpose of verification, to assess the quality of finite element calculations [24]. Its application to model registration problems was initiated for free vibration problems using natural frequencies and modes [25] and has undergone many developments using the Drucker error at first [26,27] and the dissipation error for the emphasizis of damping phenomena [28]. Since then, the efficiency of the method has been proved in transient dynamics using heavily corrupted measurements [29] and in the non linear case for visco-plasticity and rupture model updating using dynamic tests [30]. More recent work aimed at introducing stochastic information into the models to be updated [31,32]. Through a number of examples, involving both synthetic and experimental data, it was shown that the approach, which is purely mechanical, is perfectly consistent. Let us note that many other authors have relied on the good convexity properties of the CRE (or similar) residual for solving inverse problems in mechanics $[19,33,34]$ including several applications for identifying distributed variables in linear elasticity [35-38], but also in other fields such as electrostatics [39] or acoustics [40].

This paper presents an extension of the CRE-based updating method for comparing a set of in situ measurements to a family of time-dependent FE models submitted to non-stationary excitations. As part of a collaboration with ASTRIUM-ST our objective is the validation of the European ARIANE 5 launcher's model using operational data and the fact that the excitation forces applying to a spacecraft during a flight can not be considered as 'perfectly well-known inputs' is worth mentioning. Dealing with partially unknown inputs can be problematic for the resolution of the inverse problem and the strategy we describe in this paper allows one to get around this difficulty. We will define a residual, called Modified $C R E$, capable of taking into account the slowly time-varying behavior of the structure as well as integrating the whole flight database as 'experimental reference'. This functional, built from the less reliable data of the mechanical problem, enables one to consider the uncertainties related to the model, the measurements and the inputs simultaneously. Hereafter, we present an iterative algorithm which consists in selecting the erroneous FEM parameters and correcting them to decrease the distance between tests and calculations. At the end of the algorithm, erroneous parameters sets as well as an estimate of the partially unknown excitation forces are obtained within confidence intervals associated with a given level of relative error. Since our ambition is to update models of industrial size, we finally propose an innovative strategy to compute (at low numerical cost) the elements of the second order Taylor expansion of the cost function with respect to the uncertain parameters of the problem. This last improvement enables a considerable saving of computations to perform minimization and helps determine the confidence intervals.

In a first example, we illustrate using synthetic data how supplying the gradient and Hessian improves the minimization algorithm without deteriorating the quality of the confidence intervals. A second illustration implements measurements from an ARIANE 5 flight to update a unidimensional space launcher model.

\section{The CRE-based updating method}

\subsection{Reference problem |}

Let us consider a structure, within a domain $\Omega$, at time $t$ in $\left[t_{i}, t_{f}\right]$ as shown on Fig. 1. Displacements $\underline{u}^{d}$ are prescribed on the boundary $\partial_{u} \Omega$, surface loads $f^{d}$ are imposed on $\partial_{f} \Omega$, whereas body forces $\mathrm{f}$ are acting throughout the volume $\Omega$.

The reference problem consists in finding the displacements $\underline{u}(\underline{m}, t) \in \mathcal{U}^{\left[t_{i}, t_{f}\right]}$ and stresses $\underline{\underline{\sigma}}(\underline{m}, t) \in \mathcal{S}^{\left[t_{i}, t_{f}\right]}$ satisfying:

- initial conditions

- kinematic boundary conditions

$$
\forall \underline{m} \in \partial_{u} \Omega, \quad u_{i}=u_{i}^{d}
$$

- equilibrium equations

$$
\begin{aligned}
& \forall \underline{m} \in \partial_{f} \Omega, \quad \sigma_{i j} \cdot n_{j}=f_{i}^{d} \\
& \forall \underline{m} \in \Omega, \quad \rho \frac{d^{2} u_{i}}{d t^{2}}-\frac{\partial \sigma_{i j}}{\partial m_{j}}+\mathrm{f}_{i}=0
\end{aligned}
$$

- constitutive equations

$$
\forall \underline{m} \in \Omega, \quad \underline{\underline{\sigma}}=\mathbb{K}: \underline{\underline{\varepsilon}}(\underline{u})+\mathbb{D}: \underline{\underline{\dot{\varepsilon}}}(\underline{u})
$$

Following a Kelvin-Voigt approach, we define the classical Hooke tensor $\mathbb{K}$ and introduce dissipation by means of tensor $\mathbb{D}$. Subsequently, $\underline{\sigma}^{e}$ and $\underline{\sigma}^{d}$ will denote respectively the elastic and dissipative parts of the stress tensor so that $\underline{\underline{\sigma}}=\underline{\underline{\sigma}}^{e}+\underline{\underline{\sigma}}^{d}$.

Remark 1. At this point, it is important to note that the parameters defining the model behavior of the domain may not be constant over time. For space launch vehicles, the evolution of mass during a flight plays a role of great significance. The stiffness of some components containing gases at non-constant pressure may also vary with time.

\subsection{Hypothesis}

The previous remark leads us to an important assumption which will be made throughout this study: the behavior of the structure will be considered to be vibratory and slowly time-dependent. At time $t$, several modes may occur in an interval $\Omega_{t}=\left[\omega_{t}^{\min } \omega_{t}^{\max }\right]$ so that any vector $\underline{x}(t)$ defining the mechanical states of the system can be written

$\underline{x}(t)=\int_{\Omega t} \underline{X}_{t, \omega} e^{+i \omega \cdot t}+\underline{X}_{t, \omega}^{*} e^{-i \omega \cdot t}$

where the pair $\left(\omega, \underline{X}_{t, \omega}\right)$ containing frequency and complex modulus will be assumed to vary slowly with time.

\subsection{The measurements}

In the framework of model updating, we now need to introduce the measurements acquired by the sensors into the problem defined above. For this purpose, let there be two new domains:

- $\partial_{\tilde{u}} \Omega \subset \partial \Omega$, where some components of the field $\underline{u}$ or its derivatives are measured, giving access to a temporal family of Fourier pairs that we note $\left(\omega, \underline{U}_{t, \omega}\right)_{t}$,

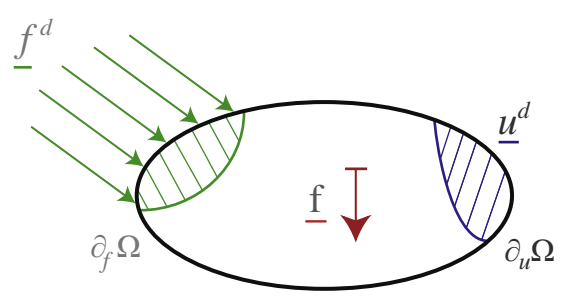

Fig. 1. Reference problem. 
- $\partial_{\tilde{f}} \Omega \subset \partial_{f} \Omega$, on which an estimate of the excitation forces denoted $\left(\omega, \underline{\tilde{F}}_{t, \omega}\right)_{t}$ is accessible.

One should note that the mechanical problem is now overspecified (ill-posed) on the possibly overlapping boundaries $\partial_{\tilde{u}} \Omega$ and $\partial_{\tilde{f}} \Omega$.

\subsection{Problem reformulation: the Modified CRE}

The major point of our approach then consists in relaxing the satisfaction of the less reliable data in the definition of spaces of admissible solutions. We separate the quantities or relations that we consider 'reliable', from those considered as 'unreliable'. Table 1 gives an example of such a separation, which can vary depending on the problem.

A solution $s=\left(\underline{U}, \underline{\underline{\sigma}}^{e}, \underline{\underline{\sigma}}^{d}, \underline{F}\right)$ verifying the reliable quantities and equations is declared 'admissible'. Let $\mathbf{S}_{t, \omega}^{a d}$ name the admissible subspace of solutions. An energy residual $e_{t, \omega}^{2}$ is defined on the less reliable data and writes for each $(t, \omega)$

$e_{t, \omega}^{2}(s)=\xi_{t, \omega}^{2}(s)+\frac{r}{1-r}\left\{\left\|\Pi_{\tilde{u}} \underline{U}-\underline{\widetilde{U}}_{t, \omega}\right\|^{2}+\left|\Pi_{\tilde{f}} \underline{F}-\underline{\widetilde{F}}_{t, \omega}\right|^{2}\right\}$

The functional is made of two terms: the CRE $\xi_{t, \omega}^{2}$ and a distance to the measurements. The model error $\xi^{2}$ relies on two mechanical concepts:

- the Constitutive Relation Error associated with elastic behavior

$$
\xi_{e l}^{2}=\frac{1}{2} \int_{\Omega}\left(\underline{\underline{\sigma}}^{e}-\mathbb{K}: \underline{\underline{\varepsilon}}(\underline{U})\right): \mathbb{K}^{-1}:\left(\underline{\underline{\sigma}}^{e}-\mathbb{K}: \underline{\underline{\varepsilon}}(\underline{U})\right) d \Omega
$$

- the dissipation error

$$
\xi_{\text {diss }}^{2}=\frac{1}{2} \int_{\Omega}\left(\underline{\underline{\sigma}}^{d}-\mathbb{D}: \underline{\underline{\dot{\varepsilon}}}(\underline{U})\right): \mathbb{D}^{-1}:\left(\underline{\underline{\sigma}}^{d}-\mathbb{D}: \underline{\underline{\dot{\varepsilon}}}(\underline{U})\right) d \Omega
$$

and is written as a linear combination of expressions (7) and (8).

$\Pi_{\tilde{u}}\left(\right.$ resp. $\left.\Pi_{\tilde{f}}\right)$ are projection operators, which when applied to vector $\underline{U}$ (resp. $\underline{F}$ ) gives the value of the vector at sensors position. $\|\cdot\|^{2}$ and $|\cdot|^{2}$ are energy error measures homogeneous to $\xi_{t, \omega}^{2}(s)$ and equivalent in level. The coefficient $r /(1-r)$ is a weighting factor which enables one to assign a greater or lesser degree of confidence to the measurements: $r$ is chosen close to 1 if the measurements are considered to be very reliable and close to 0 in the opposite case. The currently used value is 0.5 .

In summary, we seek a solution that is admissible and verifies the less reliable equations and quantities as closely as possible. The problem to solve for each pair $(t, \omega)$ then writes

$\left(P_{t, \omega}\right) \mid \begin{gathered}\text { find } \hat{s}=\left(\underline{U}, \underline{\underline{\sigma}}^{e}, \underline{\underline{\sigma}}^{d}, \underline{F}\right) \in \mathbf{S}_{t, \omega}^{a d} \\ \text { minimizing } e_{t, \omega}^{2}(s) \text { with } s \in \mathbf{S}_{t, \omega}^{a d}\end{gathered}$

Table 1

\begin{tabular}{|c|c|c|}
\hline & Model data and Eq. & Experimental data \\
\hline Reliable & $\begin{array}{l}\text { - Geometry } \\
\text { - Kinematic b.c. (1) } \\
\text { - Equilibrium Eqs. (2), (3) }\end{array}$ & $\begin{array}{l}\text { - Identified frequencies } \omega \\
\text { - Sensors position and } \\
\text { direction on } \partial_{\tilde{u}} \Omega \\
\text { - Sensors position and } \\
\text { direction on } \partial_{\tilde{f}} \Omega\end{array}$ \\
\hline Unreliable & $\begin{array}{l}\text { - Parameters of the constitutive } \\
\text { equations ( } 4 \text { ) }\end{array}$ & $\begin{array}{l}\text { - Measured disp. amplitudes } \\
\widetilde{\widetilde{U}}_{t, \omega} \\
\text { - Measured force } \\
\text { amplitudes } \underline{\widetilde{F}}_{t, \omega}\end{array}$ \\
\hline
\end{tabular}

Separation of the quantities.
Once the solution $\hat{s}$ is computed, the value of $e_{t, \omega}^{2}(\hat{s})$ called Modified CRE can be evaluated and integrated with time and frequency to include the experimental database as a whole

$J=\int_{t_{i}}^{t_{f}} \int_{\omega_{t}^{\min }}^{\omega_{t}^{\max }} e_{t, \omega}^{2}(\hat{\boldsymbol{s}}) d \omega d t$

The global error $J$ measures the correlation between the model and the test results and optimal parameters are the ones minimizing this cost function. If the measurements contribution to error is significant and associated with a small set of sensors, these sensors can be considered to be defective and excluded from the calculation of the Modified CRE.

\section{Discrete formulation of the problem $\left(P_{t, \omega}\right)$}

The updating method is developed in a FE framework which requires the problem to be discretized. The way the problem $\left(P_{t, \omega}\right)$ is solved is detailed in this section where the subscripts $t, \omega$ are dropped for simplicity's sake.

\subsection{Modified CRE}

The discretization of the problem leads to the construction of stiffness, mass and damping matrices $\mathbf{K}, \mathbf{M}$ and $\mathbf{D}$ as well as nodal vectors: the kinematically admissible displacement field $\underline{U}$ (verifying Eq. (1)) classically introduced by the FE approach and two auxiliary displacement vectors $\underline{V}$ (resp. $\underline{W}$ ) associated with the elastic (resp. dissipative) part of the stress tensor through a change of variables. Referring to (7) and (8) the model error can be expressed in its discrete form as

$$
\xi_{t, \omega}^{2}=\frac{\alpha}{2}(\underline{U}-\underline{V})^{*}[\mathbf{K}](\underline{U}-\underline{V})+\frac{\beta}{2}(\underline{U}-\underline{W})^{*}\left[T \omega^{2} \mathbf{D}\right](\underline{U}-\underline{W})
$$

where $T$ is a time parameter chosen to be proportional to $1 / \omega .(\alpha, \beta)$ are constant weighting coefficients within $[0 ; 1]$ and $\underline{A}^{*}$ stands for the transposed conjugate of $\underline{A}$.

The choice of the error measures $\|\cdot\|^{2}$ and $|\cdot|^{2}$ is not critical but must be dimensionally compatible with $\xi^{2}$. Two matrices $\left[\mathbf{G}_{\tilde{u}}\right]$ and $\left[\mathbf{G}_{\tilde{f}}\right]$ are introduced to yield

$$
\begin{aligned}
& \left\|\Pi_{\tilde{u}} \underline{U}-\underline{\widetilde{U}}\right\|^{2}=\frac{1}{2}\left(\Pi_{\tilde{u}} \underline{U}-\underline{\widetilde{U}}\right)^{*}\left[\mathbf{G}_{\tilde{u}}\right]\left(\Pi_{\tilde{u}} \underline{U}-\underline{\widetilde{U}}\right) \\
& \left|\Pi_{\tilde{u}} \underline{F}-\underline{\widetilde{F}}\right|^{2}=\frac{1}{2}\left(\Pi_{\tilde{f}} \underline{F}-\underline{\widetilde{F}}\right)^{*}\left[\mathbf{G}_{\tilde{f}}\right]\left(\Pi_{\tilde{f}} \underline{F}-\underline{\widetilde{F}}\right)
\end{aligned}
$$

so that $\left[\mathbf{G}_{\tilde{u}}\right]=\alpha\left[\mathbf{K}_{\tilde{u}}\right]+\beta T \omega^{2}\left[\mathbf{D}_{\tilde{u}}\right]$ and $\quad\left[\mathbf{G}_{\tilde{f}}\right]=\left[\alpha\left[\mathbf{K}_{\tilde{f}}\right]+\beta T \omega^{2}\left[\mathbf{D}_{\tilde{f}}\right]\right]^{-1}$ where the $\tilde{u}$ and $\tilde{f}$ subscripted quantities refer to the reduced damping and stiffness matrices at measurement locations (using classical reduction $[41,42])$.

\subsection{Admissible fields}

The solution $\hat{s}=(\underline{U}, \underline{V}, \underline{W}, \underline{F})$ in $\mathbf{S}^{a d}$ has to satisfy the equilibrium equation

$[\mathbf{K}] \underline{V}+i \omega[\mathbf{D}] \underline{W}-\omega^{2}[\mathbf{M}] \underline{U}=\underline{F}$

The displacement vectors $\underline{V}$ and $\underline{W}$ (derived from $\underline{\underline{\sigma}}^{e}$ and $\underline{\underline{\sigma}}^{d}$ ) are searched verifying the dynamic equilibrium and are said dynamically admissible. The functional $\xi^{2}$ measures the gap in the constitutive law, in other words, a distance between the kinematically and dynamically admissible displacement fields. 


\subsection{Constrained minimization problem}

The Modified CRE $e_{t, \omega}^{2}$ residual can be minimized under admissibility condition by introducing Lagrange multipliers. Let us write the Lagrangian $L(s)$ that will be of valuable use hereafter

$L(s)=e_{t, \omega}^{2}(s)+\underline{\lambda}^{*}\left\{[\mathbf{K}] \underline{V}+i \omega[\mathbf{D}] \underline{W}-\omega^{2}[\mathbf{M}] \underline{U}-\underline{F}\right\}$

Expressing the stationarity of the Lagrangian with respect to the four fields contained in $s$ leads to the inversion of the following system

$$
[\mathcal{A}] \cdot\left\{\begin{array}{l}
\underline{U} \\
\underline{\lambda} \\
\underline{F}
\end{array}\right\}=\left\{\begin{array}{c}
\frac{r}{1-r} \Pi_{\tilde{u}}^{*}\left[\mathbf{G}_{\tilde{u}}\right] \underline{\tilde{U}} \\
0 \\
\frac{r}{1-r} \Pi_{\tilde{f}}^{*}\left[\mathbf{G}_{\tilde{F}}\right] \tilde{f}
\end{array}\right\}
$$

where $\underline{\lambda}=\alpha(\underline{U}-\underline{V})=i \beta(\underline{U}-\underline{W})$ and

$\mathcal{A}=\left[\begin{array}{ccc}\frac{r}{1-r} \Pi_{\tilde{u}}^{*}\left[\mathbf{G}_{\tilde{u}}\right] \Pi_{\tilde{u}} & {\left[\alpha[\mathbf{K}]-i \beta\left[T \omega^{2} \mathbf{D}\right]-\omega^{2}[\mathbf{M}]\right]} & 0 \\ {\left[-\omega^{2} \mathbf{M}+i \omega \mathbf{D}+\mathbf{K}\right]} & -\left[\frac{1}{\alpha}[\mathbf{K}]+\frac{\omega}{\beta}[\mathbf{D}]\right] & -\Pi_{f}^{*} \\ 0 & -\Pi_{f} & \frac{r}{1-r} \Pi_{\tilde{f}}^{*}\left[\mathbf{G}_{\tilde{f}}\right] \Pi_{\tilde{f}}\end{array}\right]$

$\Pi_{f}$ is a projection operator which when applied to $\underline{\lambda}$ gives the value of the vector at nodes corresponding to an excitation area. One may observe that choosing $\alpha=1, \beta=1$ and $T=1 / \omega$ leads to a Hermitian matrix.

\section{Implementation of the updating method}

The initial model configuration is described by a given set of parameters that may be uncertain such as Young's moduli, thicknesses, damping parameters or quantities related to the excitation forces. These parameters are arranged in a vector $\underline{\theta}$ and the corresponding space is denoted $\boldsymbol{\Theta}$. The minimization problem writes

$$
\begin{gathered}
\text { find } \underline{\hat{\theta}} \in \mathbf{\Theta} \text { minimizing } \\
J_{(\underline{\theta})}=\int_{t_{i}}^{t_{f}} \int_{\omega_{t}^{\min }}^{\omega_{\max }^{\max }} e^{2}\left(\hat{\boldsymbol{S}}_{(\underline{\theta})}\right) d t d \omega
\end{gathered}
$$

where the solution $\hat{s}$ is sought in $\mathbf{S}^{a d}$. Optimal parameters $\underline{\hat{\theta}}$ are obtained recursively as the limit of a sequence $\left(\underline{\theta}^{(p)}\right)_{p}$ in an iterative localization/correction algorithm detailed below.

\subsection{Activation of the procedure}

Once the defective sensors have been eliminated from the calculation, one has to determine whether the model needs to be updated.

For each iteration $p$, we build a denominator $\mathcal{D}_{p}$

$$
\begin{aligned}
\mathcal{D}_{p}= & \frac{\alpha}{2}\left(\underline{U}^{(p)}+\underline{V}^{(p)}\right)^{*}[\mathbf{K}]\left(\underline{U}^{(p)}+\underline{V}^{(p)}\right)+\frac{\beta}{2}\left(\underline{U}^{(p)}+\underline{W}^{(p)}\right)^{*}\left[T \omega^{2} \mathbf{D}\right] \\
& \times\left(\underline{U}^{(p)}+\underline{W}^{(p)}\right)
\end{aligned}
$$

a relative error $\varepsilon_{p}^{2}$

$\forall p, \quad \forall \underline{\theta} \in \boldsymbol{\Theta} \quad \varepsilon_{p}^{2}(\underline{\theta})=\frac{e^{2}(\underline{\theta})}{\mathcal{D}_{p}}$

and the updating process is launched if

$\varepsilon^{2}\left(s_{0}\right) \geqslant \varepsilon_{0}^{2}$

where $\varepsilon_{0}^{2}$ is a given threshold value. One shall notice that once index $p$ is fixed, the denominator $\mathcal{D}_{p}$ no longer depends on $\underline{\theta}$. The threshold value $\varepsilon_{0}^{2}$ depends on the quality of the model chosen to describe the structure. For a complex and rich model capable of representing the main phenomena involved, the value can be low (typically $\leqslant 1 \%$ ). For simple, less accurate models, seeking low relative error levels is meaningless; $\varepsilon_{0}^{2}$ will be set in the interval [1;5\%]. The updating procedure is iterative and stops as soon as criterion (21) is not met.

\subsection{Localization step}

Assuming that the global model can be divided into several substructures $E \in \mathbf{E}$, the local indicator $\xi_{E}^{2}$ is calculated at each iteration $p$ for each substructure using

$\xi_{E}^{2}=\frac{\alpha}{2}\left(\underline{U}_{E}-\underline{V}_{E}\right)^{*}\left[\mathbf{K}_{E}\right]\left(\underline{U}_{E}-\underline{V}_{E}\right)+\frac{\beta}{2}\left(\underline{U}_{E}-\underline{W}_{E}\right)^{*}\left[T \omega^{2} \mathbf{D}_{E}\right]\left(\underline{U}_{E}-\underline{W}_{E}\right)$

so that $\xi^{2}=\sum \xi_{E}^{2}$. The selection of the 'most erroneous' substructures is based on the criterion

$\xi_{E}^{2} \geqslant h_{R} \cdot \max _{E \in \mathbf{E}} \xi_{E}^{2}$

with, e.g., $h_{R}=0.8$. The subset containing the substructures to be updated (usually one or two actualized at every iteration) and the associated design parameters will be denoted respectively $\mathbf{E}_{R}^{(p)}$ and $\underline{\theta}^{(p)}$.

\subsection{Correction step}

We now aim at minimizing the value of $J_{(\theta)}$ with respect to a reduced set of parameters $\underline{\theta}^{(p)}$ that has been identified in the previous step. This problem is non-linear with respect to $\underline{\theta}^{(p)}$ and we solve it using a recursive BFGS [43-46] minimization algorithm. A classical implementation of this algorithm consists in initializing the Hessian to identity so that the first iteration is equivalent to the gradient algorithm. The subsequent iterations $k$ will refine the approximation $\mathcal{H}_{k}$ of the Hessian matrix $\nabla^{2} J$. The stiffness, mass and damping matrices are reassembled and problem $\left(P_{t, \omega}\right)$ is solved for each variation of the parameters.

\subsection{Iteration of the algorithm}

Once the minimum of the cost function is reached for the subset $\mathbf{E}_{R}^{(p)}$, the functional $J$ is recomputed, a new subset $\mathbf{E}_{R}^{(p+1)}$ can be identified and the associated parameters corrected. At each iteration, new erroneous substructures can appear as a result of the substructures from the previous stages being corrected.

\subsection{End of the procedure}

If the relative error falls below the required criterion $\varepsilon_{0}^{2}$, the updating process terminates. As a result of this process, one thus obtains a sequence of subspaces $\left(\mathbf{E}_{R}^{(p)}\right)$ associated with corrected design parameters $\left(\hat{\hat{\theta}}^{(p)}\right)_{p}$ and the BFGS algorithm offers an estimate of the Hessian matrix $\left(\mathcal{H}^{(p)}\right)_{p}$ of the cost function.

The approximation of residual $J$ by its second order Taylor polynomial about the particular operating point $\underline{\hat{\theta}}^{(p)}$ leads to

$J(\underline{\theta}) \approx \widehat{J}^{(p)}+\delta \underline{\theta}^{T} \cdot \nabla J^{(p)}+\frac{1}{2} \delta \underline{\theta}^{T} \cdot\left[\hat{\mathcal{H}}^{(p)}\right] \cdot \delta \underline{\theta}$

with $\delta \underline{\theta}=\underline{\theta}-\underline{\hat{\theta}}^{(p)}$. One can easily solve the following inequality

$J(\underline{\theta}) \leqslant \mathcal{D}_{p} \cdot \varepsilon_{0}^{2} \quad \Rightarrow \quad \underline{\theta} \in I_{p}$

and give, for each iteration $p$, the value of the parameter $\underline{\theta}$ inside an interval $I_{p}$ associated with the chosen threshold value. From an updating point of view, the intervals $I_{p}$ have more sense than the optimal parameter values $\underline{\hat{\theta}}^{(p)}$ because any perturbation introduced into the measurements would affect the optimal sequence $\left(\underline{\hat{\theta}}^{(p)}\right)_{p}$. Providing optimal parameters inside confidence intervals is an original and effective way to deal with the Lack Of Knowledge associated with the numerical model as well as excitation levels. 


\subsection{Inverse problem regularization: discussion}

The major drawback minimizing quadratic cost functions (as in Eqs. (12) and (13)) is generally bad stability properties, in the sense that small data errors induce large errors in the solution. The subject of regularization, which consists of defining techniques for restoring stability, is covered by a large literature (see e.g. $[18,47])$. In this subsection, we explain how our approach leads to a regularization of the inverse problem.

A classical strategy for regularizing inverse problems is the socalled Tikhonov regularization where stability is ensured by adding to the cost function $J(\underline{\theta})$ a distance to a reference configuration. No such term is introduced in this paper but the iterative strategy we adopt for correcting the erroneous substructures (a small set of these for each iteration, see localization step Section 4.2) naturally favors an optimal configuration close to the initial one. Let us still notice that the CRE functional $\xi^{2}$ introduced while reformulating the ill-posed problem Section 3, can be interpreted as an energybased penalty term. The ratio $\frac{r}{1-r}$ plays the role of a classical penalty parameter and can be adjusted by the L-curve method [48].

One should finally remark that the overall stability of our approach is also enhanced by having a threshold criterion $\varepsilon_{0}^{2}$; the minimization algorithm will be stopped before seeking optimal parameters among unreasonable values or configurations. The confidence intervals give an idea of the disturbance on optimal parameters and provide insight into the trust that should be put in the final results.

\section{Numerical applications}

In this section, two applications of the method described above are presented.

\begin{tabular}{lll}
\hline \multicolumn{2}{c}{ Reference configuration } \\
\hline \hline$l$ & Total length & $9 \mathrm{~m}$ \\
$t$ & Shell thickness & $0.020 \mathrm{~m}$ \\
$\rho$ & Aluminum density & $2800 \mathrm{~kg} / \mathrm{m}^{3}$ \\
$E$ & Young's modulus & $70 \mathrm{GPa}$ \\
$\nu$ & Poisson's ratio & 0.3 \\
$\eta$ & Hysteretic damping & 0.04 \\
\hline \hline$n_{n}$ & Node number & 98 \\
$n_{d o f}$ & dof number & 574 \\
$n_{\text {Elt }}$ & Shell element number & 104 \\
\hline
\end{tabular}

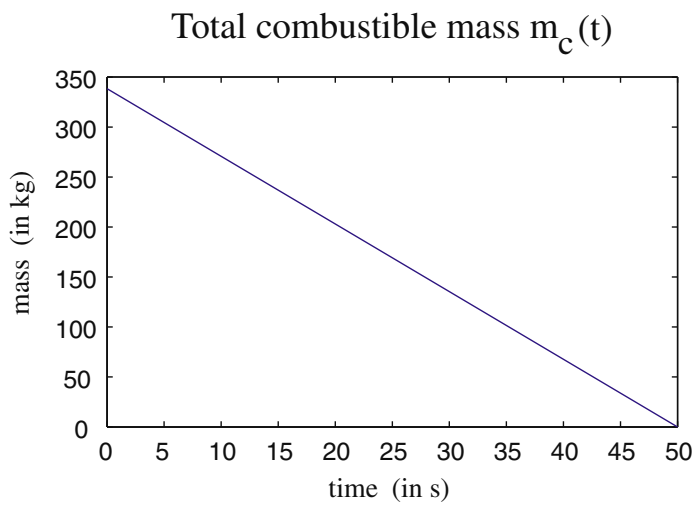

\subsection{Pressure vessel model updating}

Since the algorithm described in Section 4 was derived to deal with industrial models, the question of the effectiveness of this iterative process must be addressed. The most numerically expensive step of every iteration is the non-linear minimization of function $J_{(\underline{\theta})}$ with respect to the most erroneous parameters. Each time $J$ needs to be evaluated, the solution $\hat{s}$ has to be found and the system (16) inverted. The size of this system is around twice the number of DOFs describing the numerical model and the repetitive inversions required to perform model updating may be problematic for industrial applications. Other authors [49] have proposed a reduction technique to decrease the cost associated with the correction of large size models. In this paper, we will focus on reducing the number of $J$-evaluations which are necessary for the BFGS algorithm to reach the minimum. Our improvement, illustrated on the test case described hereafter consists of providing the algorithm with an analytical expression of the gradient and Hessian matrix of $J$.

\subsubsection{Description of the problem}

Let us consider a pressurized vessel divided into nine sub-structures as described in Fig. 2. The two upper nodes are blocked and one of the lateral nodes is retrained in displacement along the $z$ axis. $4 \%$ hysteretic damping is introduced in the model using a Kelvin-Voigt description. In addition to the aluminum shell structure, we add concentrated masses at each node to model the solid combustible whose mass $m_{c}$ is distributed on the vessel's inner walls. The time evolution of $m_{c}$ can be seen on Fig. 2. An excitation force $F$ is applied at the bottom of the structure in all directions.

The simulation results of 51 DOFs for a reference configuration $(E=70 \mathrm{GPa})$ are compared to a modified configuration where the

\section{Modified configuration}

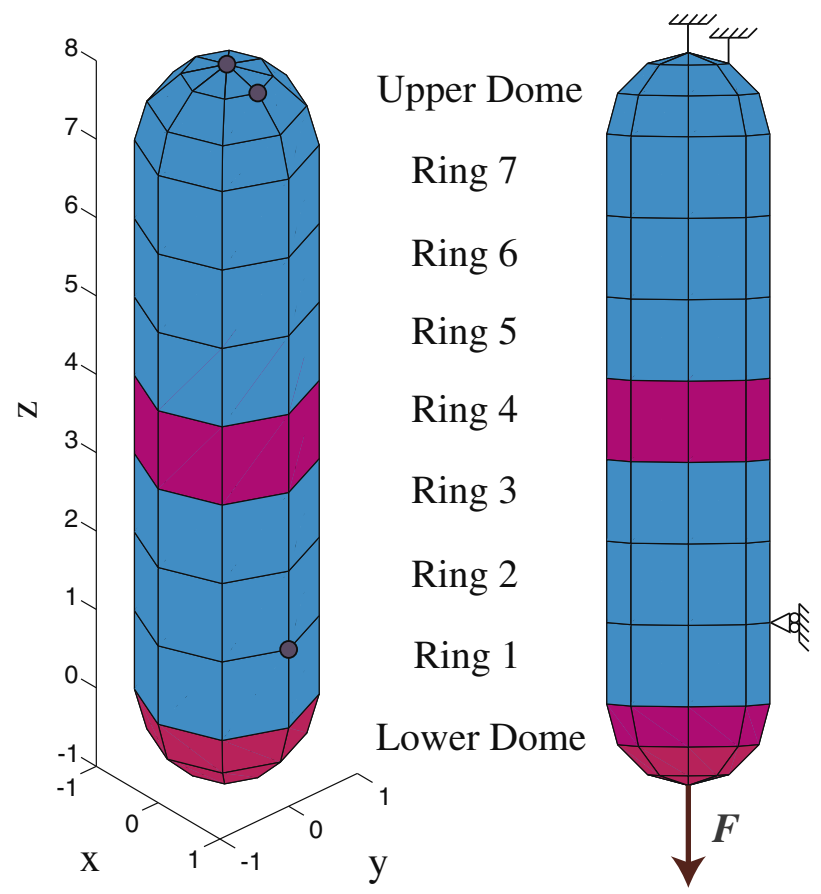

Fig. 2. Problem description - two components are modified. 
Young's modulus of two substructures (Ring 4 and Lower Dome) are changed to $E^{\prime}=80.5 \mathrm{GPa}$. Fig. 3 illustrates results given by the first iteration of the algorithm where one can see that erroneous substructures are clearly identified.

For this first calculus, referred to as Variant 1 , the gradient $\nabla \mathrm{J}$ is numerically computed and the Hessian is initialized to identity. The sequence $\left(\underline{\theta}_{k}^{(1)}\right)_{k}$ is depicted in red on Fig. 3 and an optimal solution $\hat{\hat{\theta}}^{(1)}$ is reached after 9 iterations. The results of this first calculus are summarized in Table 2 where one can notice that the routine computing the cost function $J$ has been called 39 times.

\subsubsection{Analytical expression of gradient $\nabla J$}

Let us first remark that the Modified CRE and Lagrangian $L$ (defined Eq. 15) are equal on the subspace $\mathbf{S}^{\text {ad }}$ (where the dynamic equilibrium is verified)

$\forall s \in \mathbf{S}^{a d}, \quad L(s)=e^{2}(s)$

Then, for every corrected parameter $\theta_{i} \in \underline{\theta}$

$\forall s \in \mathbf{S}^{a d}, \quad \frac{d L(s)}{d \theta_{i}}=\frac{d e^{2}(s)}{d \theta_{i}}$

which still holds for $\hat{s} \in \mathbf{S}^{a d}$ minimizing $e^{2}(s)$. The dependance of the Lagrangian to the parameters $\theta_{i}$ is implicit; its derivative consequently reads

$\forall s \in \mathbf{S}^{a d}, \quad \frac{d L(s)}{d \theta_{i}}=\frac{d L(s)}{d s} \frac{d s}{d \theta_{i}}+\frac{\partial L(s)}{\partial \theta_{i}}$

Then, observing that the solution $\hat{s}$ achieves the stationarity of the Lagrangian

$\frac{d L(\hat{s})}{d s}=0$

one can write

$\frac{d e^{2}(\hat{s})}{d \theta_{i}}=\frac{\partial L(\hat{s})}{\partial \theta_{i}}$

The partial derivatives of $L$ are computed referring to expression (15) with $\alpha=\beta=1$ to yield

$\frac{\partial L(\hat{s})}{\partial \theta_{i}}=\frac{1}{2} \underline{\lambda^{*}} \frac{\partial[\mathbf{K}]}{\partial \theta_{i}} \underline{\lambda}+\frac{T \omega^{2}}{2} \underline{\lambda}^{*} \frac{\partial[\mathbf{D}]}{\partial \theta_{i}} \underline{\lambda}+\underline{\lambda}^{*}\left\{\frac{\partial[\mathbf{K}]}{\partial \theta_{i}} \underline{V}+i \omega \frac{\partial[\mathbf{D}]}{\partial \theta_{i}} \underline{W}\right\}$
Table 2

BFGS algorithm - Stop criterion $\varepsilon_{1}=\sqrt{J / \mathcal{D}_{1}}=0.005 \%$.

\begin{tabular}{llll}
\hline Iteration $p=1$ & Variant 1 & Variant 2 & Variant 3 \\
\hline Gradient computation & Numerical & Analytical & Analytical \\
Hessian initialization & Identity & Identity & Analytical \\
Nb. of iterations $k$ & 9 & 12 & 4 \\
Nb. of $J$ evaluations & 39 & 20 & 5 \\
$\left\|\mathcal{H}_{k}-\nabla^{2} J\right\|_{F} /\left\|\nabla^{2} J\right\|_{F}$ & $25.1 \%$ & $0.006 \%$ & $0.005 \%$ \\
\hline
\end{tabular}

The integration of this last expression over time and frequency leads to the gradient of the global cost function.

The gradient finally reads as a function of the solution $\hat{s}$ and of the derivatives of matrices $\mathbf{K}$ and $\mathbf{D}$ with respect to the corrected parameters $\theta_{i}$. For complex parameters (such as thicknesses or geometry parameters), those derivatives may not be so simple to evaluate, but in any cases, especially for updating parameters with linear dependence to $F E$ matrices, the expression (31) avoids the numerical computation of the gradient as in common practice. In a second version of the calculation, referred to as Variant 2, we supply BFGS with the analytical gradient. Yielding results are collected in Table 2 for comparison.

\subsubsection{Analytical expression of the Hessian $\nabla^{2} J$}

A second improvement of the correction step can be achieved by computing the Hessian analytically. Expression (28) first needs to be differentiated with respect to $\theta_{j}$

$\frac{d}{d \theta_{j}}\left(\frac{\partial L(\hat{s})}{\partial \theta_{i}}\right)=\frac{d}{d s}\left(\frac{\partial L(\hat{s})}{\partial \theta_{i}}\right) \cdot \frac{d \hat{s}}{d \theta_{j}}+\frac{\partial^{2} L(\hat{s})}{\partial \theta_{j} \partial \theta_{i}}$

Since an analytical expression for $\frac{\partial L(\hat{S})}{\partial \theta_{i}}$ is available, writing $\frac{d}{d s}\left(\frac{\partial L(\hat{s})}{\partial \theta_{i}}\right)$ and $\frac{\partial^{2} L(\hat{s})}{\partial \theta_{j} \partial \theta_{i}}$ is an easy task. The only 'problematic' term is $\frac{d \hat{s}}{d \theta_{j}}$. However, it can be evaluated using its definition. The solution $\hat{s}$ is computed by inverting the system (16), recalled beneath in its simplified form

$$
[\mathcal{A}]\{\hat{\boldsymbol{s}}\}=\{\mathcal{B}\}
$$

$\frac{d \hat{s}}{d \theta_{j}}$ may be given by differentiating this system with respect to $\theta_{j}$

$[\mathcal{A}]\left\{\frac{d \hat{s}}{d \theta_{j}}\right\}=\left\{\frac{\partial \mathcal{B}}{\partial \theta_{j}}\right\}-\left[\frac{\partial \mathcal{A}}{\partial \theta_{j}}\right]\{\hat{s}\}$
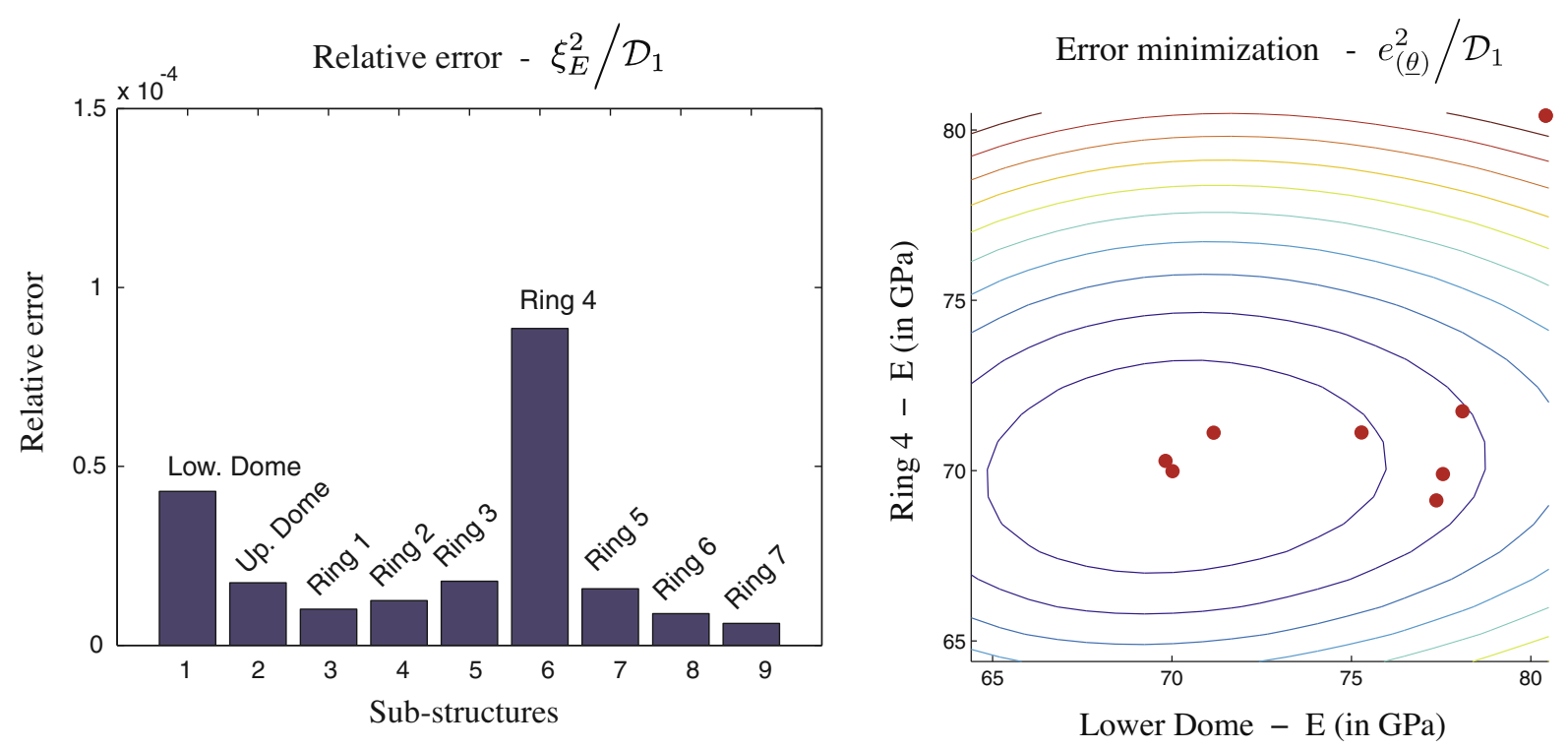

Fig. 3. Updating results - iteration $p=1-$ numerically computed gradient $-\mathcal{H}_{0}=I$. 
At this point, $\hat{s}$ has already been computed so that the right-hand side of the previous equation is entirely determined. The coefficients of the Hessian matrix are obtained after integrating expression (32) over time and frequency.

To summarize our initiative, the calculations necessary to evaluate the Hessian matrix require the right-hand side of Eq. (34) to be formed and the matrix $\mathcal{A}$ to be inverted once again, at each iteration $p$, for every time-step $t$ and frequency $f$. Knowing that a Cholesky decomposition of matrix $\mathcal{A}$ is available (since it was needed for solving Eq. (16)), evaluating $\frac{d \hat{s}}{d \theta_{j}}$ does not introduce additional time-consuming computations. However, since BFGS has the particularity of approximating the Hessian, this one can simply be initialized to its analytical value. This last strategy is implemented as Variant 3 and the corresponding results are reported in Table 2.

\subsubsection{Synthesis}

Our first improvement using an analytical expression of the gradient has led to a considerable saving of time by avoiding a numerical estimation. The number of iterations necessary to reach the minimum is similar for a given relative error threshold, but the number of calls to the cost function has decreased significantly. The routine of evaluating $J$ still needs to be called by BFGS, but this time only to refine the estimation of the Hessian matrix $\mathcal{H}_{k}$. One can finally remark that the numerical Hessian provided at the end of the algorithm in Variant 2 is of much better quality than

Table 3

Influence of a $5 \%$ noise on updating results (stop criterion and intervals in grey).

\begin{tabular}{|c|c|c|c|c|}
\hline Corrected parameters & & $E_{\text {Lower Dom }}$ & $E_{\text {Ring } 4}$ & Relative error $\varepsilon$ \\
\hline \multicolumn{5}{|c|}{ Simulated measurements without noise } \\
\hline \multirow{3}{*}{$\begin{array}{l}\text { Solution } \hat{\theta}^{(1)} \\
\text { Interval } I^{(1)}\end{array}$} & & 70.0 & 70.0 & $0.0048 \%$ \\
\hline & $\min$ & 69.7 & 70.3 & Threshold $\varepsilon_{0}$ \\
\hline & $\max$ & 69.3 & 70.7 & $0.005 \%$ \\
\hline \multicolumn{5}{|c|}{ Simulated measurements with noise } \\
\hline \multirow{3}{*}{$\begin{array}{l}\text { Solution } \hat{\theta}^{(2)} \\
\text { Interval } I^{(2)}\end{array}$} & & 70.0 & 70.1 & $0.46 \%$ \\
\hline & $\min$ & 68.9 & 71.3 & Threshold $\varepsilon_{0}$ \\
\hline & $\max$ & 68.1 & 72.1 & $0.5 \%$ \\
\hline
\end{tabular}

in Variant 1 (the relative difference in Table 2 refers to the Frobenius norm $\left.\|\cdot\|_{F}\right)$.

Our second improvement (Variant 3) which consists in initializing the Hessian to its analytical value is also worthwhile and involves 'numerical efforts' only for the first iteration $k=0$ of every step $p$. In the cases where no updating is required (i.e. low error level), the Hessian matrix calculated at the first iteration will provide direct access to the confidence intervals.

The results collected in Table 2 are obtained with an extremely low relative stop criterion $\left(\varepsilon_{0}=0.005 \%\right)$ since 'simulated measurements' were compared to the model used for generating them. Table 3 presents the more realistic values that can be expected for real tests by adding a $5 \%$ white noise one the measurements before running Variant 3.

\subsection{Simple ARIANE 5 model correction from flight data}

\subsubsection{The measurements}

The data to be operated in this analysis are taken from an ARIANE 5 flight (references not mentioned for confidentiality). The launcher is instrumented by more than 80 sensors but since the model to be updated is uniaxial (see subSection 5.2.2), only three of these sensors will be exploited as shown in Fig. 4:

- one pressure sensor at node 1 inside the combustion channel whose sample frequency is $1125 \mathrm{~Hz}$,

- two accelerometers at node 1 and 2 measuring acceleration in the vertical direction with a sample frequency equal to $446 \mathrm{~Hz}$.

In order to characterize the vibratory and slowly time dependant behavior of the structure, in accordance with the hypothesis formulated in (5), the time signals must be processed in two steps. First, a time-frequency spectrogram is computed using a Gabor transform [50] (Gaussian-like sliding windows chosen to optimize the time/frequency resolution). Then, a peak detection [51] of the spectrogram is performed, leading to the determination of $\widetilde{X}_{t, f}$ used as experimental reference. Fig. 5 shows the results we obtain by post-processing the pressure signal at section 1 .
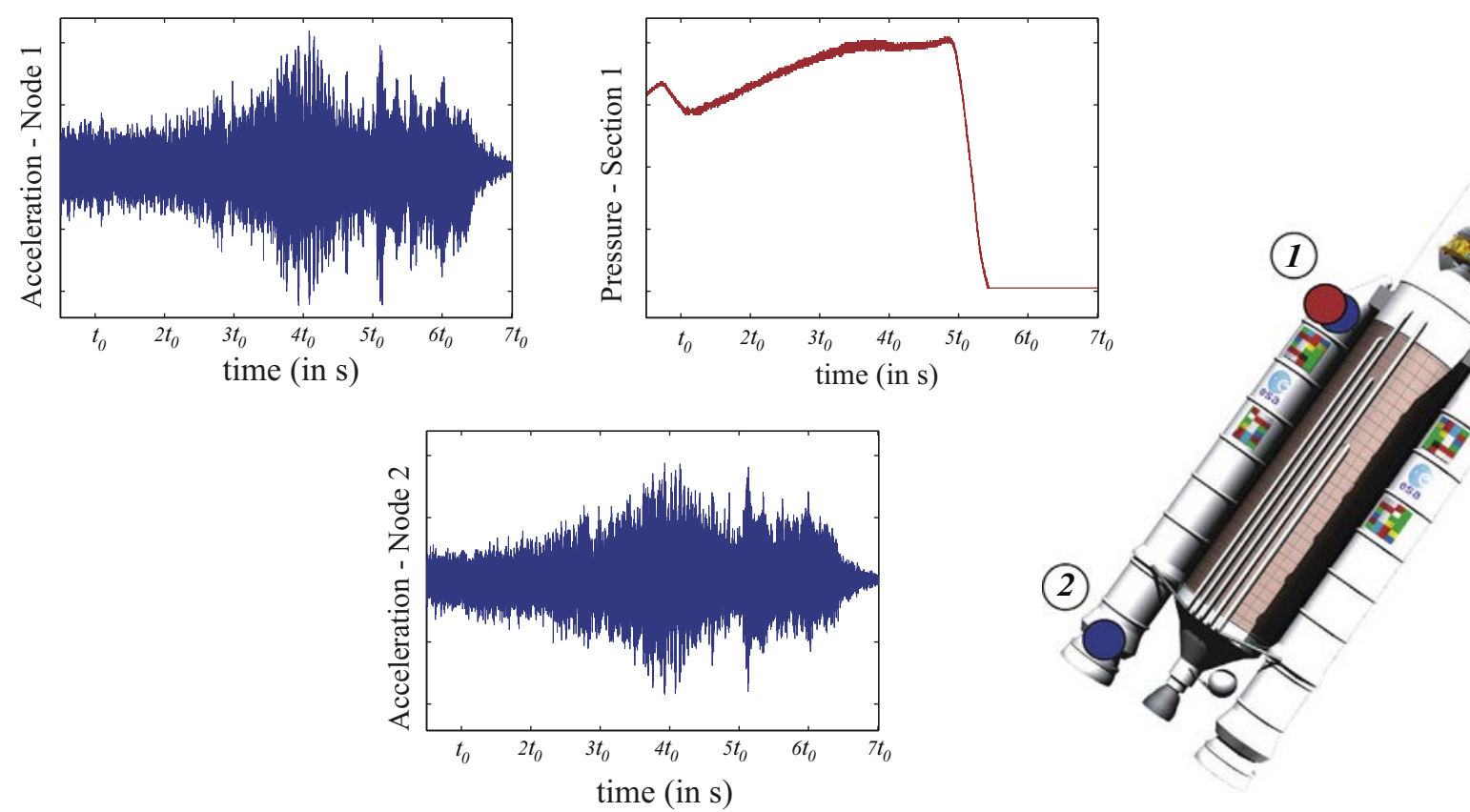

Fig. 4. Position of the sensors (hidden axis for confidentiality). 

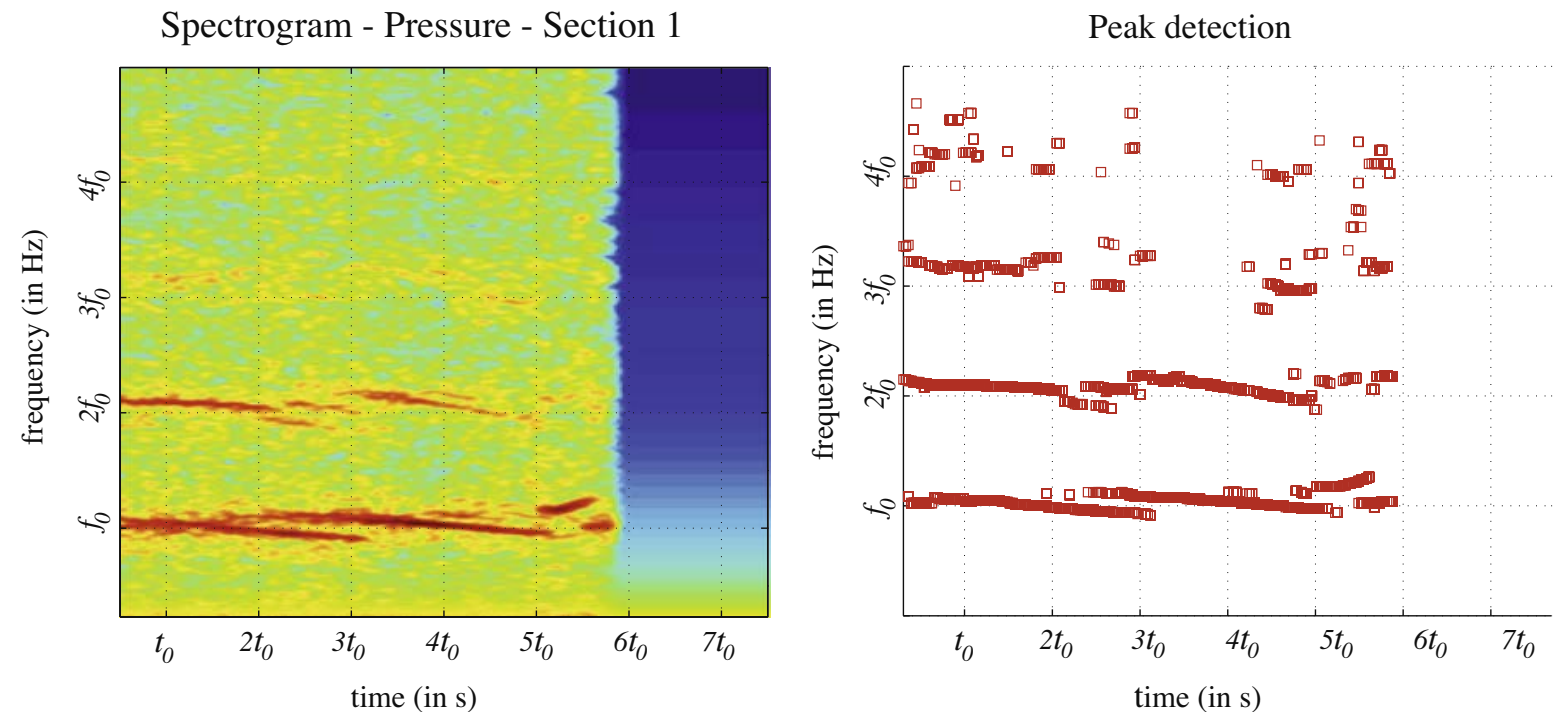

Fig. 5. Spectrogram and peak detection of the pressure sensor at Section 1.
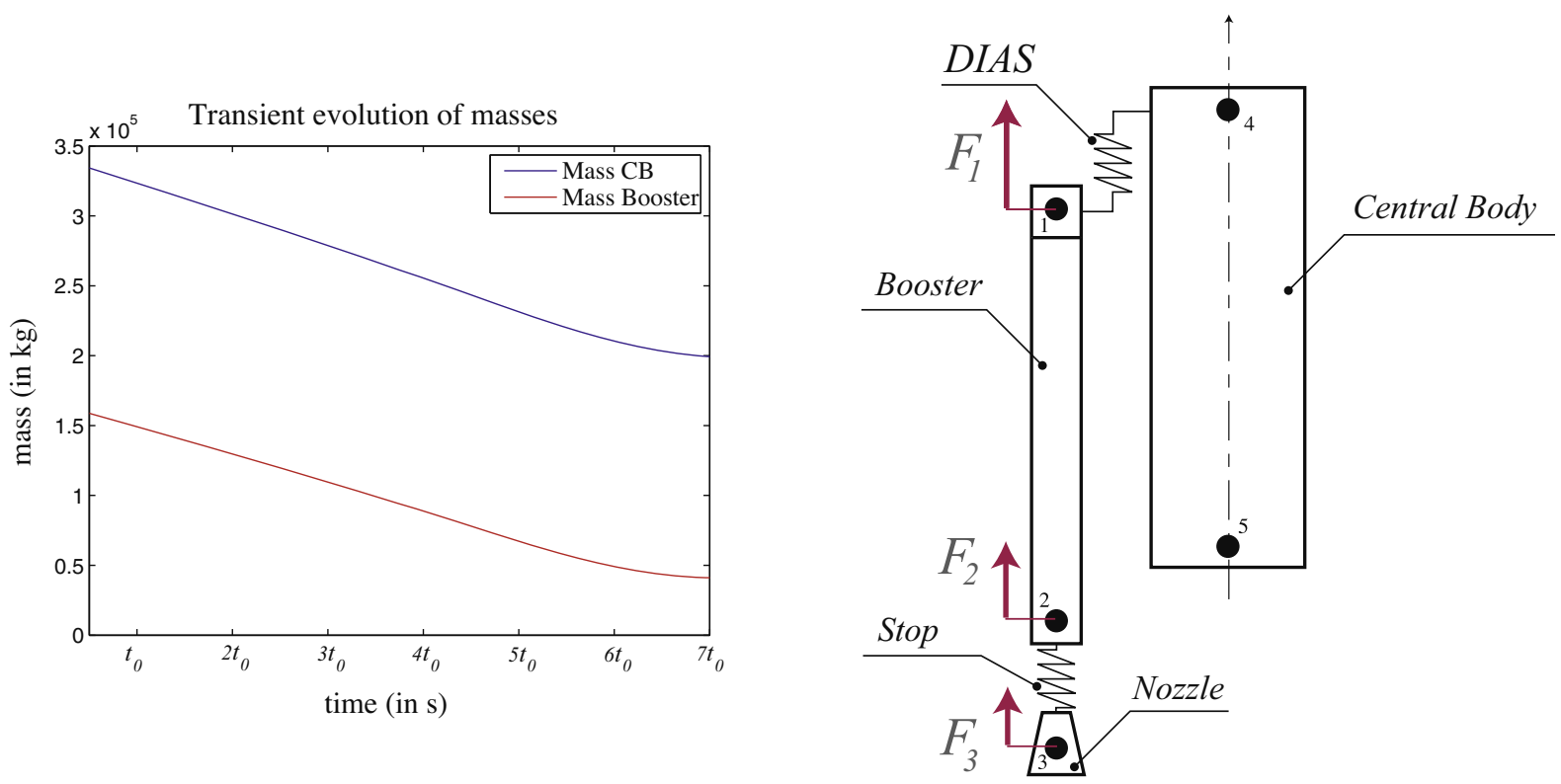

Fig. 6. Uniaxial launcher model description.

Table 4

Updating algorithm results (3\% relative error stop criterion).

\begin{tabular}{|c|c|c|c|c|}
\hline Procedure Activation & & \multicolumn{3}{|c|}{ Initial relative error $\varepsilon=6.81 \%$} \\
\hline Iteration $p=1$ & & \multicolumn{3}{|c|}{ Erroneous stiffness parameters identified } \\
\hline Corrected parameters & & $\theta_{\text {Booster }}$ & $\theta_{\text {Stop }}$ & Relative error \\
\hline Solution $\underline{\hat{\theta}}^{(1)}$ & & -0.103 & -0.240 & $3.08 \%$ \\
\hline Interval $I^{(1)}$ & $\begin{array}{l}\min \\
\max \end{array}$ & $\begin{array}{l}-0.134 \\
-0.031\end{array}$ & $\begin{array}{l}-0.291 \\
-0.141\end{array}$ & $\begin{array}{l}\text { Threshold } \varepsilon_{0} \\
3.20 \%\end{array}$ \\
\hline Iteration $p=2$ & & \multicolumn{3}{|c|}{ Erroneous damping parameters identified } \\
\hline Corrected parameters & $\eta_{\text {Booster }}$ & $\eta_{\text {Stop }}$ & Relative error & \\
\hline Solution $\underline{\hat{\theta}}^{(2)}$ & 0.082 & 0.061 & $2.87 \%$ & \\
\hline Interval $\overline{I^{(2)}}$ & $\begin{array}{l}\min \\
\max \end{array}$ & $\begin{array}{l}0.069 \\
0.090\end{array}$ & $\begin{array}{l}0.042 \\
0.078\end{array}$ & $\begin{array}{l}\text { Threshold } \varepsilon_{0} \\
3 \%\end{array}$ \\
\hline
\end{tabular}

One may note that the time-frequency spectral density of this signal is structured around four major frequencies $\left(f_{0}, 2 f_{0}, 3 f_{0}, 4 f_{0}\right)$. These frequencies correspond to acoustic modes of the gas inside the combustion channel of the booster. The existence of discontinuity areas and local peaks in the time-frequency plane is worth mentioning; those typical areas correspond to an interaction 

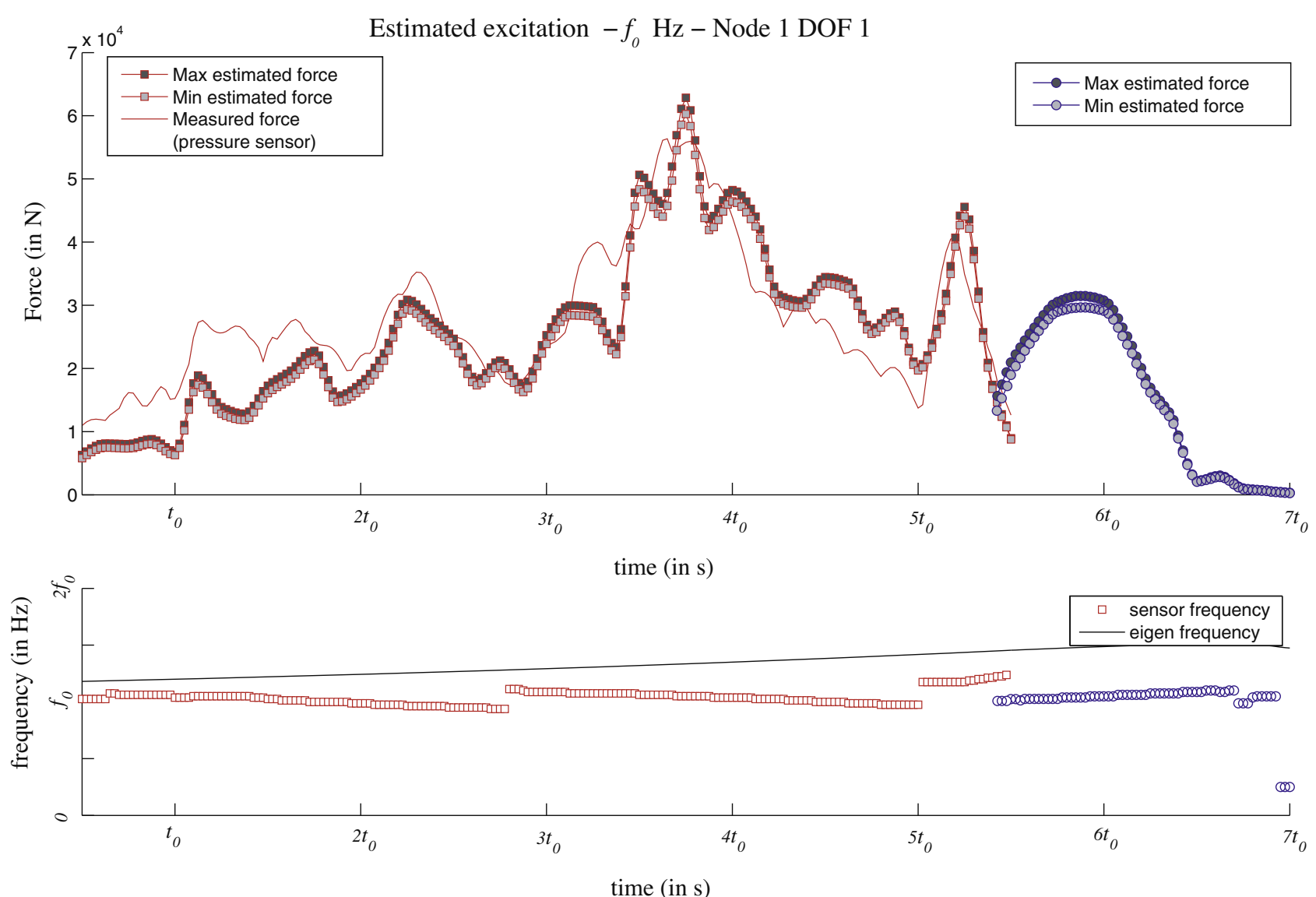

Fig. 7. Estimate of excitation corresponding to the $1^{\text {st }}$ acoustic mode.

between acoustic modes and vortex shedding characterized by Strouhal lines. For more details about those thrust oscillations, the interested reader may refer to [52,53]. These pressure oscillations determine the loading applied to the rest of the structure and govern the global vibratory response. This action is modeled by three nodal forces $F_{1}, F_{2}$ and $F_{3}$ applying on the Booster and Nozzle (see Fig. 6).

\section{Important remark}

One shall notice on Fig. 4 and 5 that the pressure sensor precludes access to excitation beyond $5.5 t_{0}$. Over the corresponding time span, the excitation forces applied to the structure are unknown.

\subsubsection{The model}

A simplified uniaxial model composed of five substructures is considered (see Fig. 6). In spite of its apparent simplicity, such a model is capable of representing the main phenomena since thrust oscillations induce a vertical loading on the launcher. Note that the slenderness of the booster is taken into account by adding 60 extra DOFs on this component. Some time dependencies such as masses evolution are also modeled and damping is introduced in the model of each component writing $\mathbf{D}_{E}=\frac{\eta_{E}}{\omega} \mathbf{K}_{E}$.

\subsubsection{Updating results}

Table 4 summarizes the results for the two iterations of the updating algorithm. The first iteration highlights modeling errors related to the booster and stop stiffnesses. Two dimmensionless parameters $\theta_{E}$ are defined such that $\mathbf{K}_{E}=\left(1+\theta_{E}\right) \cdot \mathbf{K}_{E}^{0}$ where $\mathbf{K}_{E}^{0}$ stands for the initial stiffness matrix of component $E$. Optimal parameters $\hat{\theta}$ are sought using the BFGS algorithm and confidence intervals are computed thanks to the Hessian matrix for a $3.2 \%$ relative error threshold.

\begin{tabular}{lll}
\hline & \multicolumn{2}{l}{ Reference } \\
\hline Booster & 61 DOFs & varying mass and stiffness \\
Central Body & 2 DOFs & varying mass, constant stiffness \\
DIAS & 2 DOFs & no mass, constant stiffness \\
Stop & 2DOFs & no mass, constant stiffness \\
Nozzle & 1 DOFs & nodal mass \\
5 substructures & 64 DOFs & $\eta_{E}=0.05$ \\
\hline
\end{tabular}

The second iteration identifies hysteretic damping parameters $\eta_{\text {Booster }}$ and $\eta_{\text {Stop }}$ as being erroneous and proposes new values and intervals. The process is stopped after two iterations since the error level falls below the $3 \%$ threshold value.

\subsubsection{Estimation of partially unknown excitations}

For each time step and frequency, a 3\% interval can be computed for the excitation level. Fig. 7 presents the input levels which are obtained at node 1 for the full atmospheric flight. Knowing that efforts applying after $5.5 t_{0}$ are inaccessible by the pressure sensor, the levels depicted (with blue circles) in the time interval $\left[5.5 t_{0} ; 7 t_{0}\right]$ are published here for the first time.

\subsubsection{Simulation of the corrected model}

Finally, as a verification of our results, a time simulation of the updated model is carried out using a Newmark scheme.

Fig. 8 compares the actual and simulated signals for acceleration at node 1 . The same time-frequency analysis is performed 

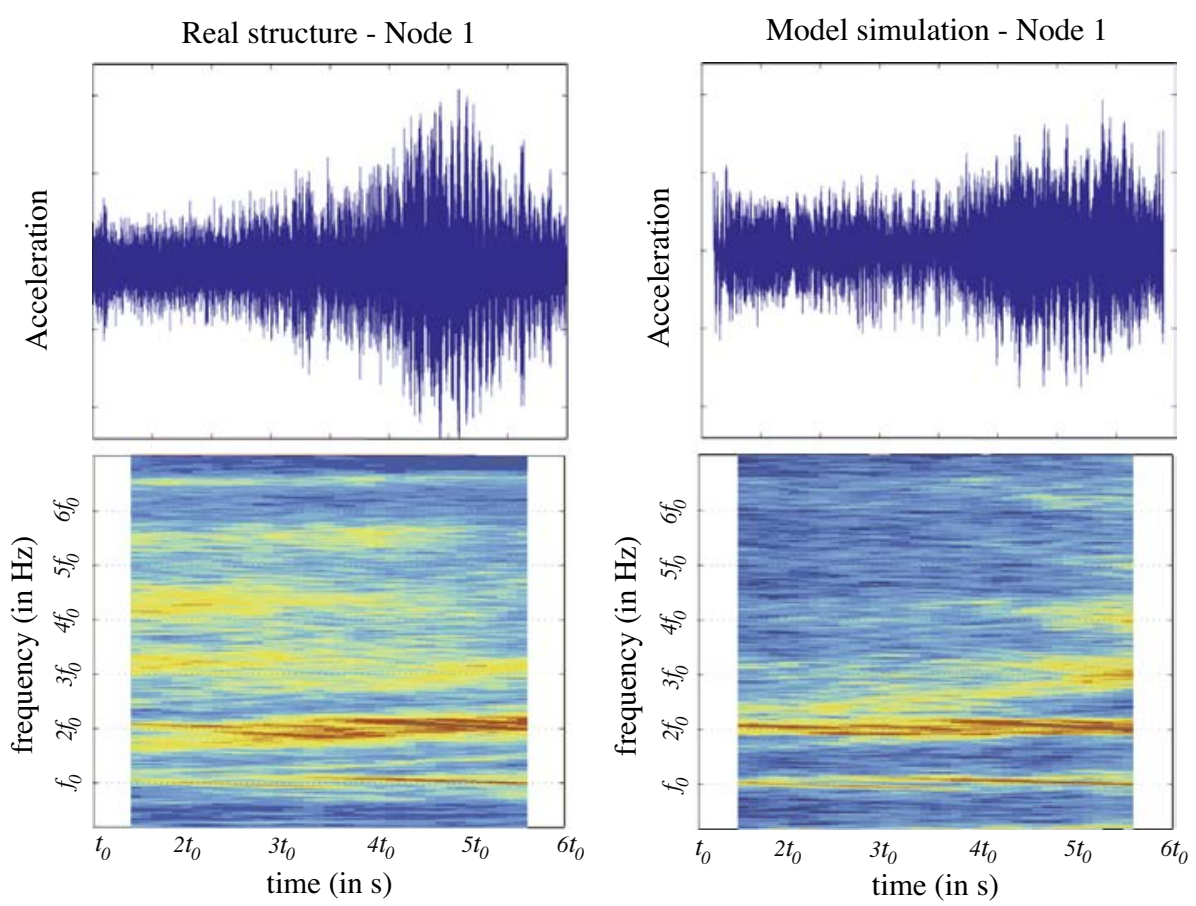

Fig. 8. Response of the actual structure (left) vs. model simulation (right).

for both signals to highlight the vibratory behavior. Actual and synthetic time histories may look different at first sight; the simple model, even corrected is not able to reproduce the spectral density of the actual structure response for high frequencies (greater than $4 f_{0}$ ) which is no surprise regarding the low modal density of our 64 DOFs model. The spectral spread is not consistent either with the actual measurements which again shows the limits of our model with respect to the many complex phenomena involved. Nevertheless, for low frequencies within $\left[0 ; 4 f_{0}\right]$, the acceleration levels and times of occurrence predicted by the corrected model are in good accordance with the measured signal. This particularly means that the updated damping levels are relevant which is worth mentioning. Thus, despite its simplicity, the updated model proves quite predictive regarding the low frequencies of the thrust oscillations $\left(f_{0}, 2 f_{0}\right.$ and $\left.3 f_{0}\right)$ which can be of interest for preliminary design and dynamic environment studies. However, the authors want to stress that updating this particular model is not an end in itself; it helped us illustrating the method on actual measurements for a non trivial case but the robustness and reliability of the approach makes it a dedicated tool for correcting models of industrial size.

\section{Conclusion}

In this paper, we have derived a robust updating algorithm suited for time-dependent industrial models along with in situ measurements. The method consists of minimizing an energy residual based on the concept of Constitutive Relation Error which leads to a reduction of the Lack Of Knowledge associated with the model and the partially unknown excitations.

The first numerical example illustrated our algorithm using synthetic measurements. Improvements were described to make it capable of handling models with a large number of degrees of freedom with reasonable computation time. We highlighted analytical expressions for the gradient and Hessian of the cost function which can be supplied to the BFGS minimization algorithm. The key point of our approach then consists in providing optimal parameters within confidence intervals associated with the low relative error values by taking advantage of the productions of the BFGS algorithm. The influence of noise on measurements has finally been studied and two typical error levels have been introduced for both 'healthy' and 'noisy' configurations.

The second example in turn, based on ARIANE 5's flight data, enabled us to illustrate the potentiality of the method using actual measurements. The updating algorithm was used to correct the parameters of a simple launcher model and enabled us to estimate some relevant partially unknown excitation levels.

\section{References}

[1] Ibrahim SR, Saafan AA. Correlation of analysis and test in modeling of structures, assessment and review. In: Proceedings of the fifth IMAC; 1987. p. 1651-60.

[2] Mottershead J, Friswell M. Model updating in structural dynamics: a survey. J Sound Vib 1993;167:347-75.

[3] Hemez FM, Doebling SW. Review and assessment of model updating for nonlinear, transient dynamics. Mech Syst Signal Process 2001;15(1):45-74.

[4] Brock JE. Optimal matrices describing linear systems. AIAA J 1968;6(7): 1292-6.

[5] Baruch M, Bar Itzhack IY. Optimum weighted orthogonalization of measured modes. AIAA J 1978;16(4):346-51.

[6] Berman A, Nagy EJ. Improvement of a large analytical model using test data. AIAA J 1983;21(8):1168-73.

[7] Kabe AM. Stiffness matrix adjustment using mode data. AIAA J 1985;23(9): 1431-6.

[8] Kammer DC. Optimum approximation for residual stiffness in linear system identification. AIAA J 1988;26(1):104-12.

[9] Smith SW, Beattie CA. Secant-method adjustment for structural models. AIAA J $1991 ; 29(1): 119-26$.

[10] Inman DJ, Minas C. Matching analytical models with experimental modal data in mechanical systems. Control Dyn Syst 1990:37:327-63.

[11] Zimmerman DC, Widengren M. Correcting finite element models using a symmetric eigenstructure assignment technique. AIAA J 1990;28(9):1670-6.

[12] Zimmerman D, Kaouk M. Eingenstructure assignment approach for structural damage detection. AIAA J 1992;30(7):1848-55.

[13] Kaouk M, Zimmerman D. Structural damage assessment using a generalized minimum rank perturbation. AIAA J 1994;32(4):836-42.

[14] Zimmerman DC, Simmermacher T. Model correlation using multiple static load and vibration tests. AIAA J 1995;33(11):2182-8.

[15] Zimmerman DC, Simmermacher T, Kaouk M. Model correlation and system health monitoring using frequency domain measurements. Struct Health Monit 2005;4(3):213-27.

[16] Zimmerman DC. Statistical confidence using minimum rank perturbation theory. Mech Syst Signal Process 2006;20:1155-72. 
[17] Antonacci E, De Stefano A, Gattulli V, Lepidi M, Matta E. Comparative study of vibration-based parametric identification techniques for a three-dimensional frame structure. Struct Control Health Monit 2012;19:579-608.

[18] Tarantola A. Inverse problem theory and methods for model parameter estimation. SIAM; 2005.

[19] Bonnet M, Constantinescu A. Inverse problems in elasticity. Inverse Probl 2005;21:R1-R50.

[20] Berger H, Ohayon R, Quetin L, Barthe L, Ladeveze P, Reynier M. Model updating methods in structural dynamics. La Recherche Aerospatiale 1991;5:9-20.

[21] Farhat C, Hemez FM. Updating finite element dynamic models using an element-by-element sensitivity methodology. AIAA J 1993;31(9):1702-11.

[22] Piranda J, Lallement G, Cogan S. Parametric correction of finite element models by minimization of an output residual: improvement of the sensitivity method. In: IMAC IX, SEM. Firenze, Italy; 1991. p. 363-8.

[23] Lammens S, Brughmans M, Leuridan J, Heylen W, Sas P. Application of a frf based model updating technique for the validation of a finite element models of components of the automotive industry. In: Design engineering technical conferences. Boston; 1995. p. 1191-1200. ASME Conferences.

[24] Ladevèze P. Leguillon D. Error estimate procedure in the finite element method and application. SIAM J Numer Anal 1983;20(3):485-509.

[25] Ladevèze P, Reynier M. A localization method of stiffness errors for the adjustment of FE models. Chapter FE modeling and analysis. ASME Publishers; 1989. p. 355-361.

[26] Ladevèze P, Chouaki A. A modelling error estimator for dynamic model updating of damped structures. In: Dulikravich GS, Tanaka M, editors. Inverse problems in engineering mechanics. Londres: Elsevier; 1998. p. 187-96.

[27] Ladevèze $P$, Chouaki A. Application of a posteriori error estimation for structural model updating. Inverse Prob 1999;15:49-58.

[28] Chouaki A, Deraemaeker A, Ladeveze P, Le Loc'h S. Model updating using the error in the constitutive relation: real case study. In: IMAC XVIII, SEM. San Antonio, Texas; 2000. p. 140-6.

[29] Allix O, Feissel P, Thévenet P. A delay damage mesomodel of laminates under dynamic loading: basic aspects and identification issues. Comput Struct 2003;81:1177-91.

[30] Nguyen HM, Allix O, Feissel P. Application of the cre for parameter identification in nonlinear dynamics with corrupted measurements. In: ICAM, editor. Seventh world congress on computational mechanics. California; 2006.

[31] Ladevèze P, Puel G, Deraemaeker A, Romeuf T. Validation of structural dynamics models containing uncertainties. Comput Methods Appl Mech Eng 2006;195:373-93.

[32] Faverjon B, Ladevèze P, Louf F. Validation of stochastic linear structural dynamics models. Comput Struct 2009;87:829-37.

[33] Balmès E. Review and evaluation of shape expansion methods. In: IMAC XVIII, SEM. San Antonio, Texas; 2000.

[34] Pascual R, Golinval JC, Razeto M. On the reliability of error localization indicators. In: ISMA 23, vol. 23. Leuven, Belgium; 1998. p. 1119-27.
[35] Constantinescu A. On the identification of elastic moduli from displacementforce boundary measurements. Inverse Prob Eng 1995:1 :293-315.

[36] Charalambopoulos A, Gintides D, Kiriaki K. The linear sampling method for the transmission problem in three-dimensional linear elasticity. Inverse Prob $2002 ; 12: 547-58$

[37] Geymonat G, Pagano S. Identification of mechanical properties by displacement field measurement: a variational approach. Meccanica 2003;38: 535-45.

[38] Florentin E, Lubineau G. Identification of the parameters of an elastic material model using the constitutive equation gap method. Comput Mech 2010;46:521-31.

[39] Kohn R, McKenney A. Numerical implementation of a variational method for electric impedance tomography. Inverse Prob 1990;6:389-414.

[40] Decouvreur V, Bouillard Ph, Deraemaeker A, Ladeveze P. Updating 2d acoustic models with the constitutive relation error method. J Sound Vib 2004;278: 773-87.

[41] Guyan RJ. Reduction of stiffness and mass matrices. AIAA J 1965;3(2):380.

[42] Irons BM. Structural eigenvalue problems: elimination of unwanted variables. AIAA J 1965;3(5):961-2.

[43] Broyden CG. The convergence of a class of double-rank minimization algorithms. J Inst Math Appl 1970;6:76-90.

[44] Fletcher R. A new approach to variable metric algorithms. Comput J $1970 ; 13: 317-22$

[45] Goldfarb D. A family of variable metric updates derived by variational means. Math Comput 1970:24:23-6.

[46] Shanno DF. Conditioning of quasi-newton methods for function minimization. Math Comput 1970;24:647-56.

[47] Tikhonov AN, Arsenin VY. Solutions of ill-posed problems. Winston and Sons; 1977.

[48] Hansen PC. Rank-deficient and discrete ill-posed problems. Philadelphia, USA: SIAM; 1998.

[49] Deraemaeker A, Ladeveze P, Leconte Ph. Reduced bases for model updating in structural dynamics based on constitutive relation error. Comput Methods Appl Mech Eng 2002;191:2427-44.

[50] Gabor D. Theory of communication. J Inst Electr Eng 1946;93(3):429-57.

[51] Delprat N, Escudié B, Guillemain P, Kronland-Martinet R, Tchamitchian P, Torrésani B. Asymptotic wavelet and gabor analysis: extraction of instantaneous frequencies. IEEE Trans Inf Theory 1992;38:644-64.

[52] Fabigon Y, Dupays J, Avalon G, Vuillot F, Lupoglazoff N, Casalis G, et al. Instabilities and pressure oscillations in solid rocket motors. Aerosp Sci Tech 2003; 7:191-200.

[53] Ballereau S, Godfroy F, Gallier S, Orlandi O, Thepenier J, Robert E, Cesco N. Evaluation method of thrust oscillations in large srm - application to segmented srm's. In: 47th AIAA/ASME/SAE/ASEE joint propulsion conference \& exhibit. San Diego, California; 2011. 